\title{
An Expedited Predictive Distributed Antenna System Based Handover Scheme for High-Speed Railway
}

\author{
Wael Ali, Junyuan Wang, Huiling Zhu, and Jiangzhou Wang \\ School of Engineering and Digital Arts, University of Kent, Canterbury, CT2 7NT, United Kingdom \\ Emails: \{wwa2, jw712, h.zhu, and J.Z.Wang\}@kent.ac.uk
}

\begin{abstract}
High-speed train has drawn considerable attention and become one of the most preferable conveyance mechanism. Each year the manufacture corporations reach a higher speed record which is expected to attain $1000 \mathrm{~km} / \mathrm{h}$ by 2021 using hyperloop one technology. Moving at such a high speed results in a high handover (HO) rate which makes it challenging for high speed railway (HSR) mobile wireless communication to preserve steady link performance. Employing distributed antenna systems (DASs) along with the two-hop architecture, this paper proposes a fast predictive $\mathrm{HO}$ algorithm. In this strategy, the serving cell starts the $\mathrm{HO}$ preparation phase in advance by inferring the train current location. Issuing the HO preparation phase in advance reduces the $\mathrm{HO}$ latency and reduces the $\mathrm{HO}$ command failure probability as well. Lower HO command failure probability means lower $\mathrm{HO}$ failure probability which could greatly improve the end-users quality of services $(\mathrm{QoS})$. The analytical results show that the proposed scheme performs better compared with the conventional HO scheme.
\end{abstract}

Index Terms-Mobile Relay, Distributed Antenna System, Handover, Frequency Switch, High-speed Railway.

\section{INTRODUCTION}

Currently, more and more attempts have been performed to satisfy the ever-growing desire for internet access due to the trending application which connects people all over the globe. This kind of popularity results in wide diverse requirements which range from simple web browsing to mobile video communication, e.g. video conferencing. Lately, high-speed railway (HSR) mobile communication system has paid a lot of attention on providing internet access with high quality of service (QoS) [1] to entice more travelers. With the global tendency towards green environment, the gradual prosperity of high-speed railway (HSR) will make it one of the most leading transportation means in the near feature. Yet, the current available mobile broadband wireless communication technology is only suitable for low-to-medium-mobility scenarios.

HSR broadband wireless communication encounters challenges from time varying channel, frequency selective fading, and high penetration loss of 10-40 dB. More importantly, high moving speed leads to frequent handover $(\mathrm{HO})$. For example, an $\mathrm{HO}$ would be required every $10 \mathrm{~s}$ assuming a coverage area of $1 \mathrm{~km}$ in conjunction with a speed of $360 \mathrm{~km} / \mathrm{h}$. The frequent $\mathrm{HO}$ results in long delay, high packet loss, and high drop off rate, degrading the overall system performance.

The current HO solution can be divided into three main categories. The first category is the location based triggering using the global positioning system (GPS) signalling [2,3].
This approach results in standardization overhead and it is unreliable in the cases of poor GPS signal reception, e.g., in tunnel scenario. The second one is the dual link approach where there are two antennas $[4,5]$. One is located on the train front and the other is on the train rear. The front antenna performs the HO scheme with the target cell while the rear antenna keeps the current link with the serving cell. The third one is distributed antenna system (DAS) based approach [6]. DAS based system architecture provides a twofold target of enhancing the spectrum efficiency and the $\mathrm{HO}$ algorithm performance.

DAS network architecture specially designed for HSR broadband wireless communication consists of hundreds of remote antenna units (RAUs) deployed linearly along the rail track in one dimensional fashion as the new HSR network tends to have less inclination angel nowadays. Each RAU is a simplified radio unit used for transmission/reception. The other part is the central processing unit (CU) where signal processing is performed [7]. RAUs are connected to the CU via either a fiber link or a wireless link. Further, the CU can control RAUs up to $20 \mathrm{~km}$, and no HO is needed between RAUs controlled by the same $\mathrm{CU}$, by employing frequency switch (FSW) scheme [8], where a frequency pattern moves/switches among RAUs along with the movement of a train so that the train is always served by the same frequency. Therefore, DAS is considered as the most effective solution to deal with the frequent $\mathrm{HO}$ issue in HSR mobile broadband wireless communications. However, in order to employ this concept, some preliminaries have to be taken into account, such as dividing the train into multiple unique frequency zones which are linked to their own corresponding RAUs communicating using the same frequencies. This means that RAU coverage area is directly related to the train carriages physical length. Specifically, each frequency zone forms a small cell in the range of $200 \mathrm{~m}$ or even less in order to have an easy and smooth synchronous switching process.

Newly published results of long-term evolution (LTE) field tests in a dense urban area exhibit an $\mathrm{HO}$ failure (HOF) rate of more than $21 \%$ [9]. Transmission failure of the HO command, also known as failure of the radio resource control (RRC) connection reconfiguration (RCR) command, is the major reason behind those failure events. Based on this observation, this paper proposes a faster $\mathrm{HO}$ algorithm which aims to reduce the failure probability of RCR command, provide a seamless 


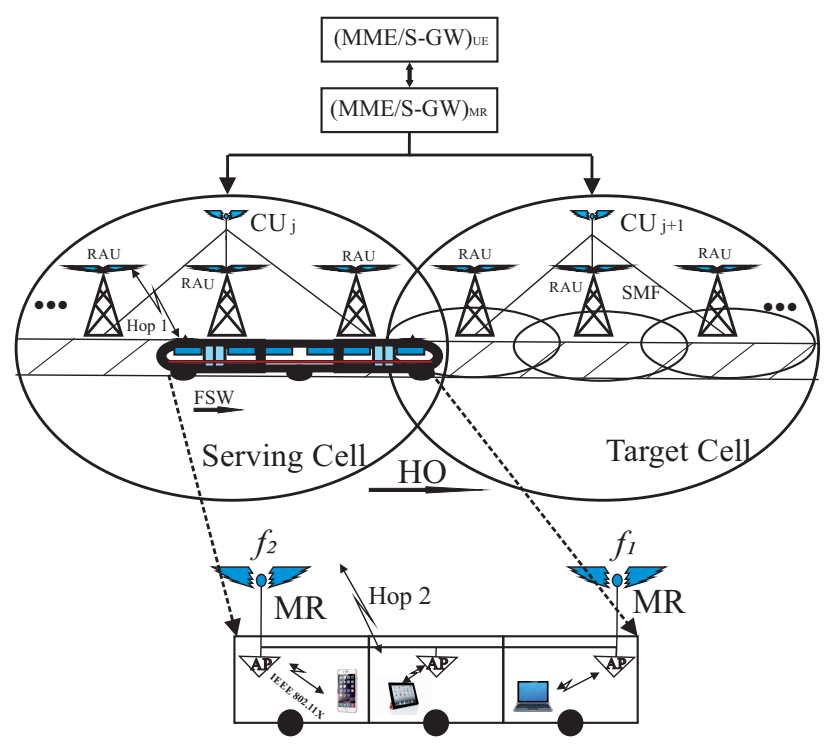

Figure 1. DAS based System architecture for high-speed railway.

service for the train's passengers by reducing the HO latency with the target of providing reliable broadband services, and considerably alleviate the effect of frequent HO. This algorithm is inspired by the property of a dedicated linear DAS network architecture, where the target cell is always the next adjacent one. Unlike the hexagonal cell distribution where the cells are distributed randomly along the rail track, this algorithm infers the train location to trigger some $\mathrm{HO}$ procedures in advance. The performance of the proposed HO scheme is evaluated when the train moves from coverage area of one RAU linking to serving CU to the coverage area of anther RAU linking to the target $\mathrm{CU}$. The proposed $\mathrm{HO}$ scheme is backward compatible with long-term evolution advanced (LTE-A) since the international union of railways has decided that the next generation standard for railway wireless communication will be based on LTE-A. The results show that our proposed scheme outperforms the traditional $\mathrm{HO}$ scheme.

The remainder of this paper is organised as follows. Section II introduces the system architecture. The proposed scheme is presented in section III. Section IV presents the HO performance analysis. Finally, section V concludes the paper.

\section{System ARChitecture}

This paper considers a two-hop architecture, i.e., RAUrelay [10] and relay-onboard, proposed by [11-13], as shown in Fig. 1. All the RAUs are deployed linearly along the rail track, which means the RAUs are deployed right at the side of the rails. By utilizing a mobile relay (MR) on the top of the train, the user equipments (UEs) communicate with the access points (APs) deployed inside each carriage, and then the APs forward the UEs packets to RAUs via MRs. This two-hop architecture is used to avoid the penetration loss of the direct link (RAU to UE and vice-versa) as well as enable simultaneous group $\mathrm{HO}$ associated with hundreds of devices that need to be handed over at the same time. Each MR represents all the UEs associated with it, therefore, the burden

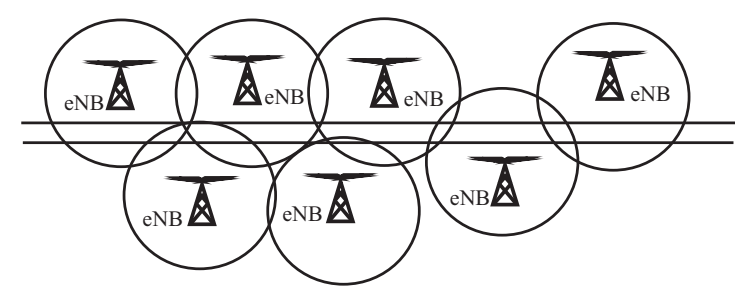

Figure 2. Conventional two-dimensional cell layout.

of the CU side will be considerably reduced during $\mathrm{HO}$ or FSW process. DAS based radio over fiber (RoF) system will be deployed linearly along the track in a one dimensional fashion, as the new HSR tracks tend to have less inclination angles, increasing the possibility of a continuous line-of-sight (LOS) connection between RAUs and MRs. In this scenario, each RAU propagates one or more non-interfering radio frequencies (RFs) and correspondingly associates with one or more RFs in the CU. This paper assumes that the train has two MRs' and correspondingly is associated with the CU at two different RFs. The core network contains a separate mobility management entity (MME) and serving/packet-gateway (S/P-GW) for MRs and UEs. Moreover, the blanket transmission strategy is utilized, where all the RAUs in the cell transmit data with equal power.

\section{The Proposed SCHEME}

In contrast to the conventional two-dimensional cell layout where there are multiple candidate target cells (see Fig. 2) located at random distance to the rail track, the linear onedimensional DAS based layout shown in Fig. 1 should lead to a more straightforward HO strategy. Therefore, taking advantage of the specialized DAS network architecture and the HSR linear track deployment is the main motivation behind the proposed HO scheme. Subsequently, this specialized dedicated architecture will result in only one candidate target $\mathrm{CU}$ which is the solely possible cell that the MR requests to $\mathrm{HO}$ to and the details of the proposed scheme are presented in the following subsection.

\section{A. Proposed Central Unit-Central Unit Handover}

In this section, an optimized fast $\mathrm{HO}$ scheme is proposed consisting of four phases: handover pre-preparation, handover preparation, handover execution, and handover completion. Fig. 3 shows the signalling flow of the traditional HO scheme, while Fig. 4 shows the signalling flow of the proposed $\mathrm{HO}$ scheme. In the following, without loss of generality, the HO process of the first MR is described. The details are as follows:

Phase I Handover Pre-preparation

1) Once the MR issues a measurement report for switching the MR from the current RAU to the next under the same serving cell $\left(\mathrm{CU}_{j}\right)$ control, $\mathrm{CU}_{j}$ detects if the target $\mathrm{RAU}$ is its last controlled RAU. $\mathrm{CU}_{j}$ could detect that easily by using either the RAU ID which is included in the measurement report sent by the MR or by the unique dedicated wavelength used to modulate the signal with (RoF based network approach). 


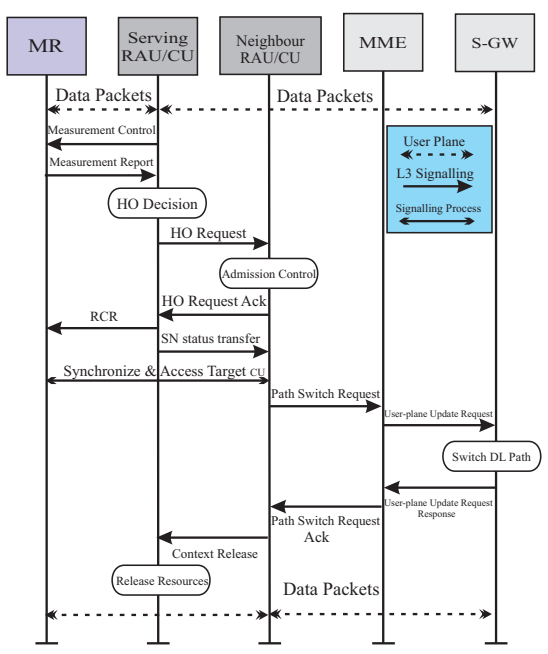

Figure 3. Conventional Central unit-central unit Handover.

2) Once confirmed, $\mathrm{CU}_{j}$ can follow one of the following approaches to trigger phase II or even use all of them cooperatively to have an accurate predicted result.

A) $\mathrm{CU}_{j}$ monitors the received frequency $f_{r}$ from the MR (received by the last RAU) until $f_{r}$ is smaller than the source frequency $f_{s}$ used by the MR $\left(f_{r}<f_{s}\right)$ and $f_{r}$ is continuously decreasing for a number of consecutive times such that $f_{r_{m+1}}>f_{r_{m+2}} \ldots>f_{r_{m+n}}$. Note that $n$ is directly related to the RAU coverage area and the maximum system speed. According to the Doppler frequency shift properties, $f_{r}>f_{s}$ when the source (i.e. MR/train) moves towards to the fixed observer (i.e. one RAU linking to $\left.\mathrm{CU}_{j}\right) ; f_{r}=f_{s}$ when the MR passes the observer; $f_{r}<f_{s}$ when the MR movies away from the observer according to the formula $f_{r}=f_{s}(1+2 v \cdot \cos \theta / c)$. When the MR is approaching, $\theta$ increases in the range of $(0, \pi / 2)$ and $\cos \theta$ decreases resulting in $f_{r}>f_{s}$ until $\cos \theta=0$. After the train is passing by the observer $\theta$ increases in the range of $(\pi / 2, \pi)$ and $\cos \theta$ decreases from zero to negative value.

B) $\mathrm{CU}_{j}$ waits for the MR to trigger the preparation phase by triggering a measurement report after detecting that the received signal strength (RSS) from target is less than the triggering threshold by some value $\alpha$ (two triggering events) or by setting an earlier triggering threshold (one triggering event).

C) $\mathrm{CU}_{j}$ can predict the MR location, for example, if we assume that the MR communicates with $\mathrm{CU}_{j}$ every 10 $\mathrm{ms}$ and $\mathrm{CU}_{j}$ can prognosticate the $\mathrm{MR}$ speed through the received signal power degradation caused by the ICI effect. The Doppler shift can be obtained according to $\Delta=1-\int_{-1}^{1}(1-$ $|x|) J_{0}\left(2 \pi f_{D} T_{s} x\right) d x$ and consequently the MR speed can be obtained. Even if the MR is changing its speed, $\mathrm{CU}_{j}$ can easily calculate that change since the train does not change its speed in a random fashion. For example, it takes 15 minutes for HSR in Taiwan to speed-up from 0 to $83.3 \mathrm{~m} / \mathrm{s}$ [14]. Also, $\mathrm{CU}_{j}$ should consider the MR's next location by taking into account the round trip time (RTT) of the system. For example, LTE system has a RTT of 70-140 ms [15].

Therefore, $\mathrm{CU}_{j}$ can predict that the MR is very close to

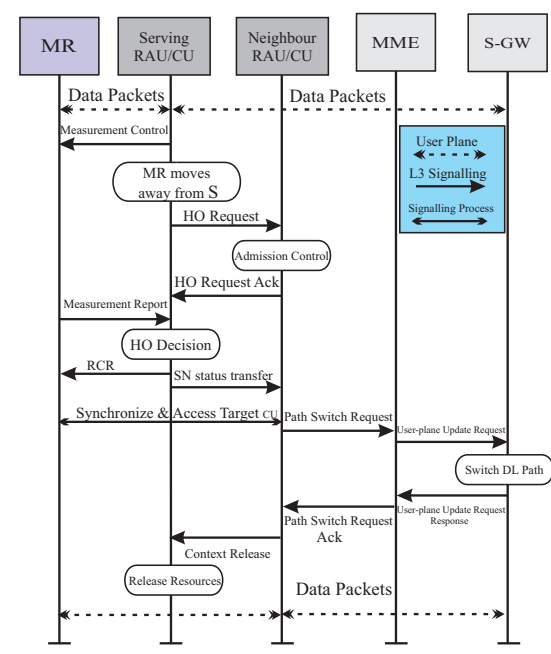

Figure 4. Proposed Central unit-central unit Handover.

the normal HO triggering event using one or all the above approaches.

Phase II Handover Preparation

3) If the condition(s) is (are) satisfied, consequently, $\mathrm{CU}_{j}$ requests the targeted neighbouring $\mathrm{CU}\left(\mathrm{CU}_{j+1}\right)$ to $\mathrm{HO}$ the most recent active UEs in advance by sending the $\mathrm{HO}$ request to $\mathrm{CU}_{j+1}$.

4) Afterwards, $\mathrm{CU}_{j+1}$ performs the admission control algorithm for the requested UEs to evaluates its ability to accept the request. If $\mathrm{CU}_{j+1}$ decides to accept the request, it sends a handover request acknowledgment (ACK) back to $\mathrm{CU}_{j}$.

5) Employing the event-triggering reporting type would guarantee a fast triggering condition. Therefore, once the RSS triggering threshold is met for a time-to-trigger (TTT) value. The MR issues the measurement report back to $\mathrm{CU}_{j}$ to trigger the process.

6) As soon as $\mathrm{CU}_{j}$ receives the measurement report, it performs the $\mathrm{HO}$ algorithm to determine whether to $\mathrm{HO}$. If $\mathrm{CU}_{j}$ decides to $\mathrm{HO}, \mathrm{CU}_{j}$ will command the $\mathrm{MR}$ to $\mathrm{HO}$ by sending RCR command to MR immediately. RCR command includes the channel access parameters required to synchronize MR with $\mathrm{CU}_{j+1}$ as well as the crucial integrity protection and ciphering. From this step and on the rest $\mathrm{HO}$ procedures are the same as the standard.

Phase III Handover Execution

7) After successfully receiving the command, the MR uses the configuration included in the command to synchronize and access $\mathrm{CU}_{j+1}$ by sending the preamble message to $\mathrm{CU}_{j+1}$. Meanwhile, $\mathrm{CU}_{j}$ sends a status transfer command to $\mathrm{CU}_{j+1}$ which initiates the data forwarding process. $\mathrm{CU}_{j+1}$ transmits the data to the MR once it becomes the serving CU.

8) If the MR successfully accesses $\mathrm{CU}_{j+1}$ by receiving the synchronization response command from $\mathrm{CU}_{j+1}$, the MR responds back with RCR complete command to confirm the success of random access procedure. Then, $\mathrm{CU}_{j+1}$ sends a path switch request command to the MME to switch the routing path of the MR. Afterwards, the MME sends a user-plane update 
Table I

SYMBOLS AND NOTATIONS.

\begin{tabular}{|ll|}
\hline Symbol & Definition \\
\hline \hline$S$ & Serving RAU \\
\hline$T$ & Target RAU \\
\hline$J_{0}()$. & Zeroth-order Bessel function of the first kind \\
\hline$T_{s}$ & Symbol duration \\
\hline$N$ & Total number of RAUs in each cell \\
\hline$P_{T}$ & Total transmitted power \\
\hline$d_{i}^{T}$ & Distance between MR and $T$ \\
\hline$d_{i}^{S}$ & Distance between MR and $S$ \\
\hline$D$ & Inter-site distance between successive RAUs \\
\hline$x_{i}$ & MR's current position \\
\hline$d_{v}$ & Vertical distance between the RAU and the track \\
\hline$f$ & Carrier frequency \\
\hline$v$ & Train speed \\
\hline$c$ & Speed of light in vacuum \\
\hline$h_{R}$ & RAU height (m) \\
\hline$h_{T}$ & Train height (m) \\
\hline$U$ & $\begin{array}{l}\text { Minimum threshold required to recover the command } \\
\text { messages successfully }\end{array}$ \\
\hline T311, T301 & $\begin{array}{l}\text { Cell reselection timer and connection reestablishment timer } \\
\text {, respectively. }\end{array}$ \\
\hline
\end{tabular}

request command to $\mathrm{S}-\mathrm{GW}$. Then, user-plane update request response is sent by S-GW back to MME, and finally MME sends a path switch request ACK command to $\mathrm{CU}_{j+1}$.

Phase III Handover Completion

9) Finally, $\mathrm{CU}_{j+1}$ sends context release command to $\mathrm{CU}_{j}$ in order to release the reserved resources associated with the MR in $\mathrm{CU}_{j}$.

\section{B. Handover Triggering Conditions}

As Fig. 1 shown, $\mathrm{CU}_{j}$ performs $\mathrm{HO}$ to neighbor $\mathrm{RAU}$ which belongs to $\mathrm{CU}_{j+1}$ if the RSS from the neighbor is above a predefined threshold for the TTT which is assumed to be zero $\mathrm{ms}$. As long as the above condition is satisfied, MR triggers the measurement report to $\mathrm{CU}_{j}$ for evaluation, and $\mathrm{CU}_{j}$ will decide whether to HO. The hysteresis parameter is not included in the above condition as the ping-pong effect [16] in this architecture will be eliminated since $\mathrm{CU}_{j}$ has a list of the current and target RAUs and the train is moving in a high-speed. In this way, a fast triggering condition that fits HSR speed as well as the RAU's small coverage area can be achieved.

Since railway is usually constructed in wide rural or viaduct areas, where multipath effect could be neglected most of the time, the main path signal is only considered [17]. With equal power allocation among RAUs, the RSS measured by the MR at the $i$ th time interval from RAU $k(k \in S, T$, where $S$ indicates the source RAU and $T$ indicates the target RAU) is denoted by $P_{i}^{k}$, and can be obtained as

$$
P_{i}^{k}[d B]=P_{t}-P_{L},
$$

where $P_{t}$ is the transmit power with a normalized noise power per RAU that includes the intercarrier interference (ICI) that resulting from the Doppler frequency shift, and can be found as follows

$$
\begin{aligned}
P_{t}[d B] & =10 \log _{10}\left(\left(P_{T} / N\right) /\left(\left(P_{T} \cdot \Delta / N\right)+1\right)\right), \\
& =10 \log _{10}\left(\frac{P_{T}}{P_{T} \cdot \Delta+N}\right),
\end{aligned}
$$

where $\Delta=1-\int_{-1}^{1}(1-|x|) J_{0}\left(2 \pi f_{D} T_{s} x\right) d x$ is the ICI power for orthogonal frequency division multiplexing (OFDM) system [18], as the system employs the LTE-A which uses OFDM as a radio interface in the downlink. $f_{D}=(v \cdot f) / c$ is the maximum Doppler frequency shift.

$P_{L}$ in (1) refers to the path loss between $S / T$ and the MR. According to D2a scenario of WINNER II model [19], $P_{L}$ can be given by

$$
\begin{aligned}
P_{L}[d B] & =44.2+21.5 \log _{10} d_{i}^{k}+20 \log _{10}(f[G H z] / 5) \\
& +10 \log _{10} g_{i}^{k}, \quad 10 m<d_{i}^{k}<d_{B R}
\end{aligned}
$$

where $d_{i}^{k}$ is the distance between the MR and the $k$ th RAU, $d_{i}^{T}$ is given by $\left(\sqrt{\left(D-x_{i}\right)^{2}+\left(d_{v}\right)^{2}}\right), d_{i}^{S}$ is given by $\left(\sqrt{\left(x_{i}\right)^{2}+\left(d_{v}\right)^{2}}\right), d_{B R}=4 h_{R} h_{T} f / c$ is the breaking point distance. Shadowing at the $i$ th time interval is represented by $\log _{10} g_{i}^{k} \sim \mathcal{N}\left(0, \sigma_{i}^{k}\right)$ that follows a Gaussian distribution with a zero mean and standard deviation $\sigma_{i}^{k}$. Now let $A[d B]=P_{t}-20 \log _{10}(f[G H z] / 5)-44.2$. Then, the RSS can be represented as

$$
P_{i}^{k}[d B]=A-21.5 \log _{10} d_{i}^{k}-10 \log _{10} g_{i}^{k} .
$$

Table I shows the symbols used in this paper and their definitions.

\section{Performance Evaluation}

\section{A. Handover Probability}

The $\mathrm{HO}$ probability is the probability that the $\mathrm{HO}$ is triggered at a position $x_{i}$. According to the HO scheme this probability can be obtained as follows

$$
\begin{aligned}
\mathbf{P} & =\mathbf{P}\left\{P_{i}^{T} \geqslant \beta\right\} \\
& =\mathbf{P}\left\{A-21.5 \log _{10} d_{i}^{T}-10 \log _{10} g_{i}^{T} \geqslant \beta\right\} \\
& =\mathbf{P}\left\{10 \log _{10} g_{i}^{T} \leqslant A-\beta-21.5 \log _{10}\left(d_{i}^{T}\right)\right\} \\
& =1-\mathbf{Q}\left(\frac{A-\beta-21.5 \log _{10}\left(d_{i}^{T}\right)}{\sigma_{i}^{T}}\right) .
\end{aligned}
$$

where $\beta$ is the triggering threshold and $Q(x)=$ $\frac{1}{\sqrt{2 \pi}} \int_{x}^{\infty} e^{\frac{-t^{2}}{2}} d t$ represents the $Q$-function. (5) also applies for the $\mathrm{HO}$ probability for the conventional $\mathrm{HO}$ scheme case. According to (5), when the MR is located at a position $x_{i}$, the $\mathrm{HO}$ process can be triggered if the detected signal strength is equal to or better than $\beta$.

\section{B. Handover Failure Probability}

$\mathrm{HO}$ probability represents the probability of HO triggering occurrence which might not end up with a successful $\mathrm{HO}$ operation. In order to obtain a successful HO all the negotiated messages through the air should be recovered correctly through 
Table II

Default Simulation Parameters.

\begin{tabular}{|ll|}
\hline Parameter & Value \\
\hline \hline Carrier Frequency & $2 \mathrm{GHz}$ \\
\hline Speed of light in vacuum & $3 \times 10^{8} \mathrm{~m} / \mathrm{s}$ \\
\hline Symbol Duration & $1 / 14 \mathrm{~ms}$ \\
\hline Total Transmit Power with normally noise power & $86 \mathrm{~dB}$ \\
\hline Shadow Fading Deviation & $4 \mathrm{~dB}$ \\
\hline Triggering Threshold $\beta$ & $-54 \mathrm{~dB}$ \\
\hline Total RAUs in each cell N & 4 \\
\hline Cell radius & $105 \mathrm{~m}$ \\
\hline Overlap & $10 \mathrm{~m}$ \\
\hline Length of Train & $400 \mathrm{~m}$ \\
\hline Distance between RAU and Track & $10 \mathrm{~m}$ \\
\hline T304, T311, T301, in ms, respectively & $50,100,1000$ \\
\hline
\end{tabular}

the indented receivers. If one of the negotiated messages is lost, the transmitter will submit the same message again until it is successfully received or reaching the maximum retransmission times. If the allowed maximum retransmission times have been reached, a radio link failure (RLF) is declared. In general, the MR negotiates five messages through the air. Two of them are with $\mathrm{CU}_{j}$ (measurement report, RCR) and the others are with $\mathrm{CU}_{j+1}$ (random access (RA), RA response (RAR), and RCR complete). Each message has a maximum number of retransmission trials specified by the standard. However, those maximum trial numbers might not even reached as the HSR might pass the overlapping area so quickly that the MR is not able to finish HO successfully within the overlapping area. This is because of the HSR harsh communication environment, i.e., the RSS is timely distorted due to Doppler frequency shift. Also, the large UE numbers that request to HO simultaneously could considerably increase the HO failure probability compared to low to medium speed scenarios. To calculate the maximum possible number of trials for HSR environment, the $\mathrm{HO}$ preparation phase is assumed to be initiated at the overlap beginning point. Then, the maximum trials for the entire HO process can be obtained as

$$
\gamma=\frac{\chi}{v \cdot\left(T_{p r e}+T_{R C R}+T 304\right)}
$$

where $\chi$ is the overlapping area, $T_{p r e}, T_{R C R}$, and T304 are the HO preparation time, the time required to recover RCR successfully, and the RA process expiry time with $\mathrm{CU}_{j+1}$, respectively (see Table III). (6) implies that $\gamma$ increases by either increasing $\chi$ or decreasing the time of the operations in the denominator. However, reducing the last two might increase the failure probability since it reduces the retransmission attempts assuming a fixed $v$. By contrast, our proposed scheme can decrease the failure probability since it reduces $T_{\text {pre }}$ by expediting the RCR triggering process which results in reducing $T_{R C R}$ duo to triggering RCR in an earlier position with a better RSS. More specifically, the RCR command is ready to be transmitted to the MR once the measurement report is received, so there is no need to send $\mathrm{HO}$ request and wait for $\mathrm{CU}_{j+1}$ response which might delay the process. Then, the maximum number of trials associated with $\mathrm{CU}_{j}$ side $(\mathrm{RCR}$

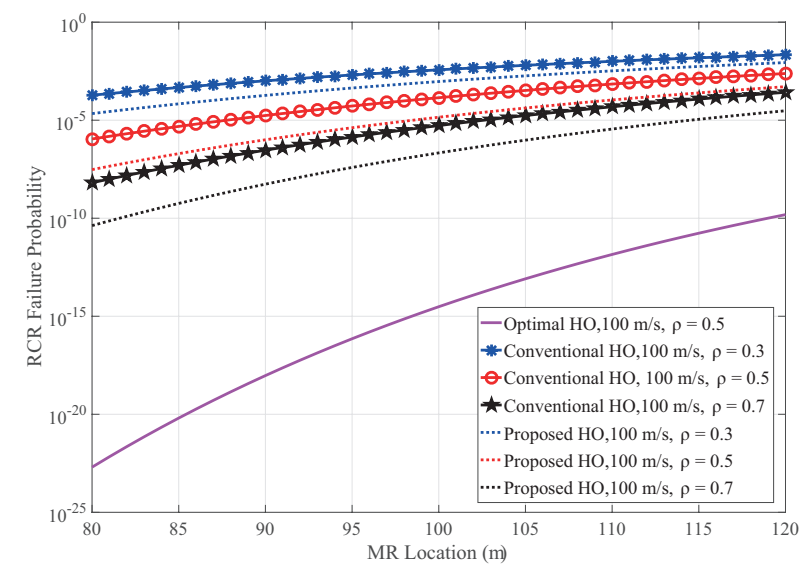

Figure 5. RCR failure probability versus the MR location under $\beta=-55 \mathrm{~dB}$, and $U=-57 \mathrm{~dB}$.

maximum trails count) is.

$$
\delta=\frac{\rho \cdot \chi}{v \cdot T_{R C R}},
$$

where $\rho$ is the percentage of the overlapping area dedicated to recover the RCR correctly. So the failure probability of RCR command reception can be found in (8) which does not consider the retransmission attempts, and (9) refers to the final RCR command reception failure probability. While the failure probability of the whole HO scheme which includes the RCR and RA procedure for only one trial can be found in (10) and (11) denotes the final HO failure probability which considers the maximum allowed trials.

The most crucial part of the HO scheme is the reception of the RCR command in an expeditious manner. Since the train is moving away from the current RAU to target RAU which implies a continuous degradation in the RSS of the serving RAU. Failing to recover RCR might lead to increased delay (due to retransmission attempts) or even a RLF situation which wastes the resources and interrupt the users' services. Therefore, receiving RCR with the best possible RSS is very critical to finish the HO scheme successfully. Once the MR receives RCR successfully, the MR tries to synchronize and access the target RAU by negotiating RA, RAR, and RCR complete commands. These command should have a lower failure probability than RCR command, since the train is moving towards the target at a high speed, so the RSS of target is getting better and better.

Fig. 5 shows the RCR failure probability. As it can be seen, as $\rho$ decreases $\mathbf{P}_{f_{T}}^{R C R}$ increases. Moreover, the proposed scheme shows a better performance compared to conventional scheme. While the optimal scheme gives the best performance, as it assumes to recover RCR command from the first transmission attempt ( $T_{R C R}$ is assumed to be $8 \mathrm{~ms}$ for one transmission trail).

Further, Fig. 6 shows the total HO failure probability. It can be seen that the optimal scheme shows the best performance, while the proposed scheme performs better than the conven- 


$$
\begin{aligned}
\mathbf{P}_{f \mid P_{i}^{T} \geq \beta}^{R C R} & =\left\{P_{i}^{S}<U\right\} \\
& =\mathbf{P}\left\{A-21.5 \log _{10} d_{i}^{S}-10 \log _{10} g_{i}^{S}<U\right\} \\
& =\left[\mathbf{Q}\left(\frac{A-21.5 \log _{10}\left(d_{i}^{S}\right)-U}{\sigma_{i}^{S}}\right)\right] . \\
\mathbf{P}_{f_{T}}^{R C R} & =\left[\left[\mathbf{Q}\left(\frac{A-21.5 \log _{10}\left(d_{i}^{S}\right)-U}{\sigma_{i}^{S}}\right)\right]^{\delta} \cdot \mathbf{P}\left\{P_{i}^{T} \geqslant \beta\right\}\right] \\
& =\left[\left[\mathbf{Q}\left(\frac{A-21.5 \log _{10}\left(d_{i}^{S}\right)-U}{\sigma_{i}^{S}}\right)\right]^{\delta} \cdot\left[1-\mathbf{Q}\left(\frac{A-\beta-21.5 \log _{10}\left(d_{i}^{T}\right)}{\sigma_{i}^{T}}\right)\right]\right]
\end{aligned}
$$

$$
\begin{aligned}
\mathbf{P}_{f}^{h} & =\left[\mathbf{P}_{f_{T}}^{R C R}+\left(1-\mathbf{P}_{f_{T}}^{R C R}\right) \cdot \mathbf{P}\left\{P_{i}^{T}<U\right\}\right], \\
\mathbf{P}_{f_{T}}^{h} & =\left[\mathbf{P}_{f_{T}}^{R C R}+\left(1-\mathbf{P}_{f_{T}}^{R C R}\right) \cdot \mathbf{P}\left\{P_{i}^{T}<U\right\}\right]^{\gamma} .
\end{aligned}
$$

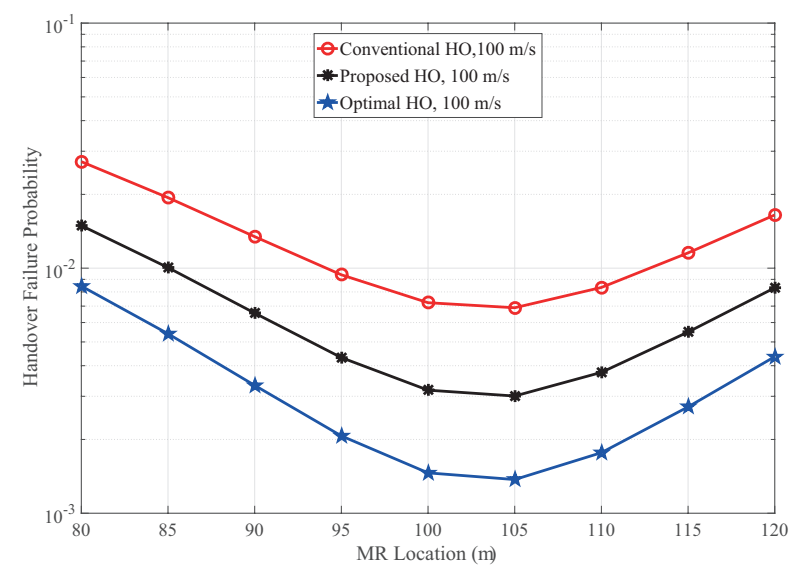

Figure 6. HOF probability versus the MR location under $\beta=-55 \mathrm{~dB}$, and $U=$ $-57 \mathrm{~dB}$.

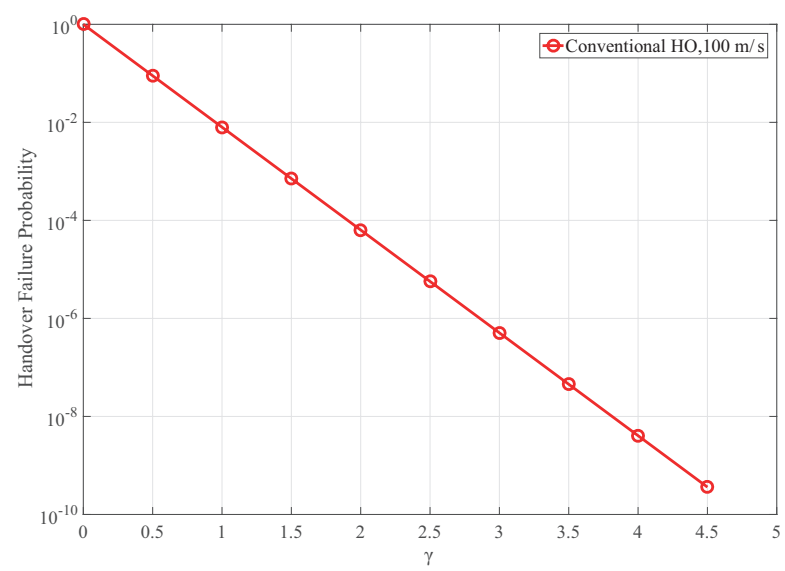

Figure 7. HOF as a function of maximum retransmission trials evaluated at the MR location of $100 \mathrm{~m}$ under $\beta=-55 \mathrm{~dB}$, and $U=-57 \mathrm{~dB}$

tional one. Moreover, Fig. 7 depicts $\mathbf{P}_{f_{T}}^{h}$ as a function of $\gamma$, as can be seen, $\mathbf{P}_{f_{T}}^{h}$ decreases as $\gamma$ increases.

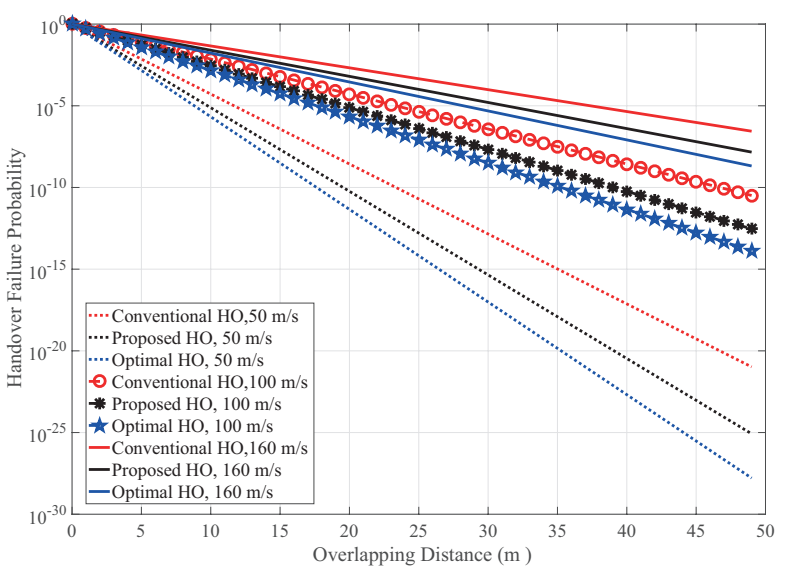

Figure 8 . HOF probability versus the overlapping length evaluated at the MR location of $100 \mathrm{~m}$ under $\beta=-55 \mathrm{~dB}$, and $U=-57 \mathrm{~dB}$.

Table III

HANDOVER LATENCY PROCESS ANALYSIS.

\begin{tabular}{|c|ccc|}
\hline HO Phases & Conventional & Proposed & Optimal \\
\hline HO decision & $16 \mathrm{~ms}$ & $9 \mathrm{~ms}$ & $16 \mathrm{~ms}$ \\
\hline HO command $\left(T_{R C R}\right)$ & $32 \mathrm{~ms}$ & $25 \mathrm{~ms}$ & $8 \mathrm{~ms}$ \\
\hline Synchronization & $50 \mathrm{~ms}$ & $50 \mathrm{~ms}$ & $50 \mathrm{~ms}$ \\
\hline Path Switch & $13 \mathrm{~ms}$ & $13 \mathrm{~ms}$ & $13 \mathrm{~ms}$ \\
\hline Total Latency $\left(T_{s}\right)$ & $111 \mathrm{~ms}$ & $97 \mathrm{~ms}$ & $87 \mathrm{~ms}$ \\
\hline
\end{tabular}

\section{Overlapping Area}

The overlapping area between successive RAUs is a crucial factor in the overall system performance design. Increasing the overlapping area guarantees a successful HO. Therefore, obtaining a sufficient overlapping area is a decisive factor to assure a stable system. Fig. 8 shows the HOF probability for different overlapping areas. It can be noticed that as the overlapping area increases the failure probability decreases, as this gives the system enough time to finish the HO successfully. Furthermore, the higher the speed the higher the failure probability and the more overlapping area required to reach the same performance. 


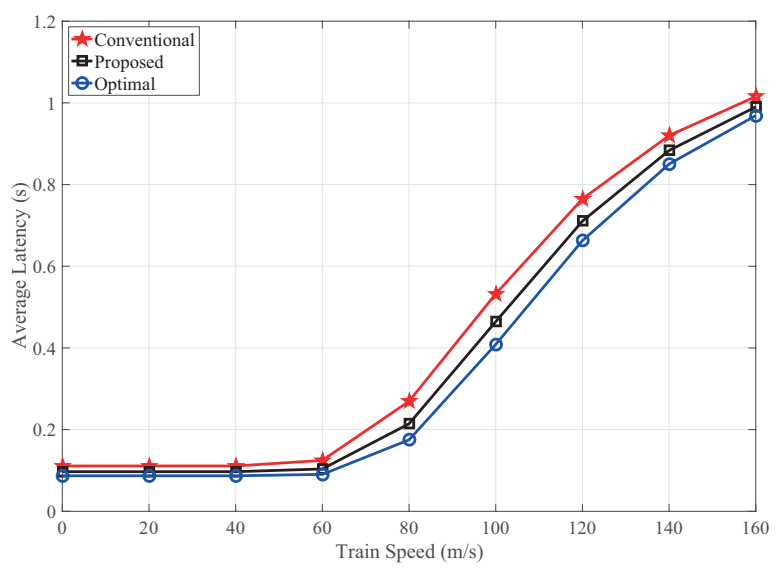

Figure 9. Handover average latency as a function of varying train speed evaluated at the MR location of $100 \mathrm{~m}$ under $\beta=-55 \mathrm{~dB}$, and $U=-57 \mathrm{~dB}$.

\section{Average Latency}

$\mathrm{HO}$ latency is another decisive performance metric when it comes to user experience and the provided QoS. The total average latency can be found as

$$
T=\left(1-\mathbf{P}_{f_{T}}^{h}\right) \cdot T_{s}+\mathbf{P}_{f_{T}}^{h} \cdot T_{r e c},
$$

where $T_{s}$ and $T_{\text {rec }}\left(T_{\text {rec }}=T 304+T 311+T 301\right.$ see Table II) are the HO latencies in case of a successful and failure situations, respectively. Fig. 9 shows the HO latency for the investigated cases. We can see that, the proposed scheme shows a comparable performance compared to the optimal one. Also, as the speed increases the latency increases. The reason is that the failure probability increases with the train speed. Further, we have used our proposed parameter $H$ to obtain Fig. 9. Which was used in [8] to equalize the effect of the train varying speed, so that varying speed will have the same HO triggering probability which can be obtained as.

$$
H=10 \log _{10} \frac{\Delta_{s}}{\Delta_{c}}
$$

where $\Delta_{s}$ (for $v=100 \mathrm{~m} / \mathrm{s}$ ) and $\Delta_{c}$ are the ICI that result from the system specific and the current HSR speeds, respectively. Therefore, the HO triggering probability for a varying speed can be obtained as follows.

$$
\begin{aligned}
\mathbf{P} & =\mathbf{P}\left\{P_{i}^{T}-H \geqslant \beta\right\} \\
& =1-\mathbf{Q}\left(\frac{A-\beta-21.5 \log _{10}\left(d_{i}^{T}\right)-H}{\sigma_{i}^{T}}\right) .
\end{aligned}
$$

Furthermore, Table III shows the HO latency of each scenario for a successful $\mathrm{HO}$. The proposed scheme shrinks the $\mathrm{HO}$ decision phase up to $56.25 \%$, and this gives the system a better chance to finish HO successfully with lower latency. Note that Table III calculation is based on [14, 20,21]

\section{CONCLUSion}

This paper proposed a specialized DAS network for future HSR wireless communication systems. The conventional HO scheme is analyzed based on this architecture. Also, an enhanced faster HO scheme is proposed. The proposed scheme was evaluated analytically in terms of failure probability, overlapping area, and average latency. Our results show that compared to conventional $\mathrm{HO}$, the proposed scheme is able to deliver application with high QoS requirement, as more reliable and seamless HO can be achieved.

\section{REFERENCES}

[1] R. Q. Hu and Y. Qian, "An energy efficient and spectrum efficient wireless heterogeneous network framework for $5 \mathrm{~g}$ systems," IEEE Communications Magazine, vol. 52, no. 5, pp. 94-101, May 2014.

[2] O. B. Karimi, J. Liu, and C. Wang, "Seamless wireless connectivity for multimedia services in high speed trains," IEEE Journal on Selected Areas in Communications, vol. 30, no. 4, pp. 729-739, May 2012.

[3] M. Cheng, X. Fang, and W. Luo, "Beamforming and positioning-assisted handover scheme for long-term evolution system in high-speed railway," IET Communications, vol. 6, no. 15, pp. 2335-2340, Oct. 2012.

[4] L. Tian, J. Li, Y. Huang, J. Shi, and J. Zhou, "Seamless dual-link handover scheme in broadband wireless communication systems for high-speed rail," IEEE Journal on Selected Areas in Communications, vol. 30, no. 4, pp. 708-718, May 2012.

[5] X. Yu, Y. Luo, and X. Chen, "An optimized seamless dual-link handover scheme for high-speed rail," IEEE Transactions on Vehicular Technology, vol. 65 , no. 10 , pp. $8658-8668$, Oct. 2016.

[6] Z. Liu and P. Fan, "An effective handover scheme based on antenna selection in ground train distributed antenna systems," IEEE Transactions on Vehicular Technology, vol. 63, no. 7, pp. 3342-3350, Sept. 2014.

[7] J. Wang and L. Dai, "Asymptotic rate analysis of downlink multi-user systems with co-located and distributed antennas," IEEE Transactions on Wireless Communications, vol. 14, no. 6, pp. 3046-3058, Jun. 2015.

[8] W. Ali, J. Wang, H. Zhu, and J. Wang, "Distributed antenna system based frequency switch scheme evaluation for high-speed railways," Accepted by IEEE Int. Conf. Commun. (ICC) 2017, Paris, 21-25 May, 2017.

[9] 3GPP RAN2 R2-140089, "Mobility Performance in Real Networks," 3rd Generation Partnership Project (3GPP), Qualcomm Incorporated, Feb. 2014.

[10] Q. Li, R. Q. Hu, Y. Qian, and G. Wu, "Cooperative communications for wireless networks: techniques and applications in lte-advanced systems," IEEE Wireless Communications, vol. 19, no. 2, Apr. 2012.

[11] B. Lannoo, D. Colle, M. Pickavet, and P. Demeester, "Radio-over-fiberbased solution to provide broadband internet access to train passengers [topics in optical communications]," IEEE Communications Magazine, vol. 45, no. 2, pp. 56-62, Feb. 2007.

[12] Y. Zhou, Z. Pan, J. Hu, J. Shi, and X. Mo, "Broadband wireless communications on high speed trains," in 2011 20th Annual Wireless and Optical Communications Conference (WOCC), Apr. 2011, pp. 1-6.

[13] J. Wang, H. Zhu, and N. J. Gomes, "Distributed antenna systems for mobile communications in high speed trains," IEEE Journal on Selected Areas in Communications, vol. 30, no. 4, pp. 675-683, May 2012.

[14] M. S. Pan, T. M. Lin, and W. T. Chen, "An enhanced handover scheme for mobile relays in lte-a high-speed rail networks," IEEE Transactions on Vehicular Technology, vol. 64, no. 2, pp. 743-756, Feb. 2015.

[15] T. Han and N. Ansari, "Radiate: radio over fiber as an antenna extender for high-speed train communications," IEEE Wireless Communications, vol. 22, no. 1, pp. 130-137, Feb. 2015 .

[16] D. Lopez-Perez, I. Guvenc, and X. Chu, "Mobility management challenges in $3 \mathrm{gpp}$ heterogeneous networks," IEEE Communications Magazine, vol. 50, no. 12, pp. 70-78, Dec. 2012.

[17] S. Jeon and S. Lee, "A relay-assisted handover technique with network coding over multihop cellular networks," IEEE Communications Letters, vol. 11, no. 3, pp. 252-254, Mar. 2007.

[18] Y. Li and L. J. Cimini, "Bounds on the interchannel interference of ofdm in time-varying impairments," IEEE Transactions on Communications, vol. 49, no. 3, pp. 401-404, Mar. 2001.

[19] P. Kysti, J. Meinil, L. Hentil, X. Zhao, T. Jms, C.Schneider, M. Narandzi, M. Milojevi, A. Hong, J. Ylitalo, V. Holappa, M. Alatossava, R. Bultitude, Y. de Jong, and T. Rautiainen, "Winner ii channel model," Techincal Report, Sep. 2007, [Available Online:http://www.ist-winner.html].

[20] 3GPP R3-120233, "Performance Analysis for Candidate Architectures Supporting Mobile Relay," 3rd Generation Partnership Project (3GPP), Sophia-Antipolis, France, Feb. 2012.

[21] 3GPP TS 36.331, "Evolved Universal Terrestrial Radio Access (EUTRA); Radio Resource Control (RRC); Protocol Specification," 3rd Generation Partnership Project (3GPP), Sophia-Antipolis,France, July. 2012. 